\title{
Borax assisted synthesis of dialdehyde Carboxymethyl cellulose-Dextrin/Gelatin based biocompatible self-healing hydrogel
}

\section{Amit Kumar Sharma}

Dr BR Ambedkar National Institute of Technology

Priya Bhalla ( $\Delta$ priya_bhalla@ymail.com )

Dr BR Ambedkar National Institute of Technology https://orcid.org/0000-0001-6547-3544

\section{Balbir Singh Kaith}

Dr BR Ambedkar National Institute of Technology

\section{Bhagya Shree}

Dr BR Ambedkar National Institute of Technology

\section{Simran Sehgal}

Dr BR Ambedkar National Institute of Technology

\section{Saiyam Arora}

Dr BR Ambedkar National Institute of Technology

\section{Research Article}

Keywords: Self-healing, imide bond, rheological studies, blood compatible

Posted Date: April 23rd, 2021

DOI: https://doi.org/10.21203/rs.3.rs-415355/v1

License: (1) This work is licensed under a Creative Commons Attribution 4.0 International License. Read Full License 


\section{Abstract}

The hydrogels with self-healing properties have high potential applications in the biomedical field. Generally, the imine bonds are used as the dynamic covalent bonding in the self-healing hydrogels and hydrogen bonding is used for providing additional strength to self-healing hydrogels. In the presented work, Gelatin, dialdehyde dextrin, and dialdehyde Carboxymethyl cellulose (CMC) based hydrogels were prepared which are biocompatible and biodegradable and hence, can be used in biomedical field. Borax and glutaraldehyde were used as binding agent and cross-linker, respectively. The dynamic imide bond formed between gelatin-dialdehyde dextrin, gelatin-dialdehyde $\mathrm{CMC}$, and gelatin-glutaraldehyde were responsible for self-healing ability of the prepared hydrogel. The rheological studies were also carried out which showed that as a consequence of lower viscosity the hydrogels can show injectable properties. The sample was also found to be blood compatible, which further highlights its biomedical importance.

\section{Introduction}

Natural hydrogels are the three dimensional form of cross-linked natural polymers which have gained importance in the present era due to their specific properties like stimuli-responsive nature, biocompatibility and biodegradable behavior significant for their biomedical use as wound dressings, in tissue engineering and drug delivery (Zhao et al., 2019).

Self-healing polymers, as the name suggests, are the networks possessing reversible interactions among the various functional groups on polymeric chains which can break on cutting and heal up through autonomous and spontaneous bonding when kept in contact for a specific period. The concept was inspired from the natural skin tissues getting self-repaired after breakage due to injuries (Tu et al., 2019; Narkar \& Lee, 2018; Shi et al., 2015). The various possibilities may include electrostatic interactions (Cao et al., 2017), dynamic chemical bonds (Maeda, Otsuka, \& Takahara, 2009), hydrogen bonds (Sherrington \& Taskinen, 2001), supra-molecular host-guest chemistry (Tuncaboylu, Sari, Oppermann \& Okay, 2011), metal (acceptor)-ligand (donor) coordinate bonding (Holten-Andersen et al., 2011) and hydrophobic interactions (Tuncaboylu, Sari, Oppermann \& Okay, 2011).

Dynamic self-healing hydrogels usually offer stimuli responsive behavior towards temperature, $\mathrm{pH}$, light and electric field (Holten-Andersen et al., 2011; Chen, Peng, Thundat., Zeng, 2019). The healing behavior is essential for various biomedical applications such as wound repair, as scaffolds for tissue engineering, in drug delivery and as injectable hydrogels (Talebian et al., 2019). Phenomenon of healing is however against the mechanical strength of polymers. Weaker interactions are responsible for more dynamic healing, which are meant to show low mechanical strength. Hence, the multi-phase systems are considered now a day which show required mechanical strength along with faster healing properties. It would resist the effects of strain applied on implantable self-healing hydrogels which may affect their functional performance along with microorganism invasion (Tu et al., 2019; Wang et al., 2019). 
Hence, there is a need to design self-healing materials in a way that they can be used for in-vivo as well as in-vitro biomedical applications. They should possess an optimum approach towards biocompatibility and tissue engineering application along with probable degradation.

Hydrogels are the gels having tendency of absorbing water and water loving species. They are 3D polymer based matrices having certain hydrophilic groups such as $-\mathrm{OH},-\mathrm{NH}_{2}$ and $-\mathrm{COOH}$ groups. Hydrogels mostly prepared from natural backbones are highly biocompatible and biodegradable in nature. They can also be prepared from physical or dynamic crosslinks and can be imbibed with selfhealing properties (Sharma et al., 2019; Priya, Kaith, Shanker, Gupta, Bhatia, 2018; Sharma, Kaith, Gupta, Shanker, Lochab, 2018; Shalviri, Liu, Abdekhodaie \& Wu, 2010; Pourjavedi, Soleyman, Bardajee \& Ghavami, 2009).

Carboxymethyl cellulose is a derivative of cellulose possessing carboxymethyl groups attached to oxygen of some of the $\mathrm{O}-\mathrm{H}$ groups present at D-glucopyranose units of chain. These units are linked by $\beta$ (1 4) linkages. Usually 0.6-0.95 groups per monomer are derivatized using different derivatization methods. $\mathrm{CMC}$ is used as thickening agent, in ice cream industries, for purification of proteins as ion exchangers and as stabilizer in emulsions of various food products (Buhus, Popa \& Desbrieres, 2009; Zhivkov \& Hristov, 2015; Hollabaugh, Burt \& Walsh, 1945). Dextrin is composed of D-glucose units linked through a (14) and a (1 6) linkages and are synthesized as a result of hydrolysis of starch and glucose. They are used to prepare emulsions, dry bandages, for mordant thickening, polishing of cereals, for making matches and other fireworks, as a product for textile coating and finishing (Alvani, Qi \& Tester, 2011).

Gelatin is translucent derivative of collagen obtained from animal body parts. A typical structure of gelatin is composed of -Ala-Gly-Pro-Arg-Gly-Glu-4Hyp-Gly-Pro- units attached in the same sequence. It consists of single or multi strands of proline left handed helix conformations extended between 50-1000 amino acids. It is used as gelling agent in various food products as well as non-food items, for the preparation of juices, as coating agent and in the preparation of easily swallowed capsule shells (Mariod \& Adam, 2013; Dakin, 1920).

Modification of these mentioned natural backbones can be done in order to synthesize self-healing and biocompatible biomaterials. In this way, various physical and dynamic covalent crosslinks can be inserted in between them (Shen et al., 2016; Maia, Carvalho, Coelho, Simões \& Gil, 2016; Pettignano, 2017; Lai et al., 2019).

Balakrishnan et al., 2019 have synthesized pH and glucose responsive in situ gelling hydrogels formed via imine and borate diol ester linkages and have utilized it for targeted drug action in tumor therapy. Khamrai et al., 2019 have carried out the ionic modification of bacterial cellulose and used it for the preparation of gelatin modified self-healing wound dressing film. Bhattacharyya et al., 2019 have prepared cytidine and boronic acids based self-healing hydrogel induced via $\mathrm{Ag}^{+}$ions. Such type of material possesses thixotropic and antimicrobial properties and can be used for stimuli responsive drug delivery. 
Presently, not much work has been carried on self-healing hydrogels as per literature. Although during past decade (2008-2018), the research articles on this particular topic have increased due to their increasing demand in various applications. The number of publications has got doubled as compared to the year 2013 publications. That shows that the topic is highly in demand and one of the currently preferred topics of research in the field of biomedical applications. In a patented work (US9533469B2), temperature responsive nanofiber based nanocomposite with healing and shape-memory effect has been designed. Other patents such as US 2014/0243428A1, WO2014176304A1 and WO2012159106A3 also provide the information about the importance of self-healing hydrogels in daily life (Tu et al., 2019).

Hence, it was decided to prepare novel highly biocompatible and biodegradable self-healing hydrogel network from modified CMC, modified dextrin and gelatin using borax and glutaraldehyde as binding and crosslinking agents, respectively. The prepared hydrogel was further tested for self-healing behavior along with its rheological properties, degradation behavior in various biological $\mathrm{pH}$ solutions and other fluids. Its blood compatibility was also tested to prove its biocompatibility.

\section{Experimental}

\subsection{Materials and methods}

Carboxymethyl cellulose (CMC), dextrin (Dex) and gelatin (Gel) were taken as primary natural backbones for the synthesis of self-healing hydrogels and were purchased from Sigma Aldrich. Other materials used were sodium periodate and ethylene glycol for the oxidation of backbones CMC and Dex which were of Sigma Aldrich brand. In addition, borax and glutaraldehyde used as binding and crosslinking agents, respectively were purchased from again Sigma Aldrich Corporation. Disodium hydrogen phosphate $\left(\mathrm{Na}_{2} \mathrm{HPO}_{4} \cdot 7 \mathrm{H}_{2} \mathrm{O}\right)$, sodium dihydrogen phosphate $\left(\mathrm{NaH}_{2} \mathrm{PO}_{4} \cdot \mathrm{H}_{2} \mathrm{O}\right)$, urea and sodium chloride were all purchased from sigma Aldrich only. Double distilled water was used for the experimental analysis of each type.

Various instrumentation techniques used for the characterization of backbones, their derivatives and final hydrogel were Fourier Transform-Infrared Spectrophotometer (Agilent Carry-630), Field Emission Scanning Electron Microscope (Nova Nano FE-SEM 450) of gold coated samples with Energy Dispersive X-ray Spectroscopy (EDS + EDS mapping) and X-ray Photoelectron Spectroscopy (ESCA ${ }^{+}$omicron nanotechnology oxford instruments). UV Spectrophotometer (Agilent Carry 100) and Rheometer (RheolabQC from Anton Paar, Austria) were used for the in vitro blood compatibility test and to study rheological properties, respectively. In addition, CPA-225D Sartius Analytical balance was used for weighing purpose and Cyberscan 1100, Eutech Instruments was used for the preparation of pH solutions.

Various reaction parameters such as weight ratio of oxidized backbones (1:1 weight ratio) to gelatin, amount of water, concentration of borax and glutaraldehyde were optimized with respect to minimum gelling time. Gelling time is the time taken by sol to get converted to gel form at room temperature (Zhao 
et al., 2019; Pettignano et al., 2017; Balakrishnan, Joshi \& Banerjee, 2013; Balakrishnan, Joshi, Jayakrishnan \& Banerjee, 2014; OU \& BO, 2017).

\subsection{Synthesis of $\mathrm{CMC}_{o x}-\mathrm{Dex}_{o x}-\mathrm{Gel}$ based self-healing hydrogel:}

\subsubsection{Dialdehyde oxidation of $\mathrm{CMC}\left(\mathrm{CMC}_{\mathrm{ox}}\right)$}

$1.0 \mathrm{~g}$ of $\mathrm{CMC}$ was dissolved in $100 \mathrm{ml}$ double distilled water. It was followed by the addition of $6 \% \mathrm{w} / \mathrm{v}$ $\mathrm{NaIO}_{4}(12 \mathrm{ml})$ and the solution was kept on stirring in dark (for $24 \mathrm{~h}$ ) at room temperature. After $24 \mathrm{~h}$, oxidation was terminated by the addition of 2-3 $\mathrm{ml}$ ethylene glycol. The dialysis of final mixture was carried out to purify the product and mixture was freeze dried afterwards to get final product. To confirm the formation of dialdehyde CMC, FT-IR spectrum of the final product was taken in the range of 500$4000 \mathrm{~cm}^{-1}$ (Lei et al., 2019).

\subsubsection{Dialdehyde oxidation of dextrin ( Dex $_{\text {ox }}$ )}

The oxidation of dextrin was carried out in the similar manner as explained under Sect. 2.2.1. The final product was dialyzed and freeze dried at the end. FT-IR spectrum of the product was considered for confirmation of dialdehyde formation of dextrin (Dex ${ }_{\mathrm{ox}}$ ) (Lei et al., 2019; Ginting, Pasaribu, Masmur, Kaban \& Hestina, 2020).

\subsubsection{Synthetic route for self-healing hydrogel}

Oxidized CMC and oxidized dextrin were dissolved in double distilled water in presence of borax on a magnetic stirrer. Similarly, gelatin was dissolved separately in double distilled water in presence of borax. Slight heating $\left(40^{\circ} \mathrm{C}\right)$ was used in case of gelatin to get a homogeneous solution. The proportion for both borax concentration and amount of water was kept same for oxidized backbones and gelatin. The solutions prepared were mixed together at room temperature on magnetic stirrer. While stirring, glutaraldehyde was added to the above mixture solution so that, sol-gel conversion takes place homogeneously. Gelling time was noted down for sol to gel conversion of the solution immediately after addition of glutaraldehyde. The final sample was washed with double distilled water to remove unreacted impurities (Shen et al., 2016; Maia, Carvalho, Coelho, Simões \& Gil, 2016; Pettignano et al., 2017).

\subsection{Self-healing behavior}

The self-healing hydrogel prepared above can be prepared in the form of various molds. Two different forms cylindrical and cubic were taken. The samples were cut into two equal halves using a cutter, kept in contact with each other and pressed together for some time. The self-healing behavior was observed after certain time (Ginting, Pasaribu, Masmur, Kaban \& Hestina, 2020; Yang, Lu, Zhang \& Zhou, 2015; Chen et al., 2017; Jalalvandi \& Shavandi, 2018; Naghizadeh, Karkhaneh \& Khojasteh, 2018).

\subsection{Rheological properties}


Rheological behavior of the synthesized hydrogel was studied in order to predict its mechanical stability. Freshly prepared cubic hydrogel of dimensions $1 \mathrm{~cm} \times 1 \mathrm{~cm} \times 1 \mathrm{~cm}$ was taken for the determination of shearingeffect and was placed under shear of parallel plate system having a diameter of $50 \mathrm{~mm}$ and separated by $1 \mathrm{~mm}$ gap and thermostat was used to maintain the temperature at $25^{\circ} \mathrm{C}$. Movable plate comes down at a certain speed called shear rate. The shear rate was set in the range of 0 to $50 \mathrm{~s}^{-1}$ and change in viscosity ( $\mathrm{mPa}$ ) as well as shear stress $(\mathrm{Pa})$ was studied as we increase the shear rate for 120s (Talebian et al., 2019; Chen et al., 2017; Zheng et al., 2018; Ouyang, Highley, Rodell, Sun, \& Burdick, 2016; Grosskopf et al., 2019; Amaral, Emamzade \& Pasparakis, 2018; Boere et al., 2015; Shen, Yang, Cui \& Cao, 2019; Stapleton et al., 2019).

\subsection{Degradation behavior}

The in vitro study for degradation of hydrogel synthesized was also carried out to ensure its applicability in biomedical applications. The sample of known weight was dipped in $100 \mathrm{ml}$ solutions such as $5 \mathrm{M}$ urea solution, phosphate buffer, $\mathrm{pH}$ solutions of acidic and basic form such as 2.93, 4.98, 7.79, 9.19 and 11.03. $5 \mathrm{~g}$ of sample of cubic form $(1 \mathrm{~cm} \times 1 \mathrm{~cm} \times 1 \mathrm{~cm})$ was dipped in $100 \mathrm{ml}$ solution of each category incubated at $37^{\circ} \mathrm{C}$ and its weight change was observed for seven days. The solution was filtered first every day and the weight of sample was note down. The \% weight change was evaluated using the formula given in Eq. 1:

$\%$ Weight change $=\frac{\text { Initial Weight }- \text { Final Weight }}{\text { Initial weight }} \times 100$

For each solution, three parallel e readings were taken and an average value was considered as the final $\%$ weight change (Lei et al., 2019).

\subsection{Blood compatibility test}

For the possible use of synthesized hydrogel in biomedical applications, it was undergone for blood compatibility test. $4 \mathrm{ml}$ of fresh blood sample was taken and it was kept preserved immediately after adding $4 \mathrm{ml}$ of $3.8 \mathrm{w} / \mathrm{v}$ EDTA solution. Hydrogel sample of cubic form $(1 \mathrm{~cm} \times 1 \mathrm{~cm} \times 1 \mathrm{~cm})$ was taken and placed in $7 \mathrm{ml}$ of normal saline solution with $0.9 \mathrm{w} / \mathrm{v} \mathrm{NaCl}$ solution. Normal saline ( $0 \%$ hemolysis) and distilled water ( $100 \%$ hemolysis) were considered as negative and positive control, respectively. The sample containing normal saline, $7 \mathrm{ml}$ of distilled water and $7 \mathrm{ml}$ of saline solution were taken in three different sterile centrifuge tubes. All of them were incubate at $37^{\circ} \mathrm{C}$ for $24 \mathrm{~h}$. After $24 \mathrm{~h}, 1 \mathrm{ml}$ of blood sample was added to each solution and kept under incubation for $1 \mathrm{~h}$ each. After $1 \mathrm{~h}$, all the samples were centrifuged for 10-15 minutes to settle down the insoluble hemoglobin. The supernatant was taken and tested under UV-visible Spectrophotometer. The absorbance value with respect to wavelength at 540 $\mathrm{nm}$ was considered for the calculation of hemolysis ratio given by Eq. 2 (Lei et al., 2019): 


\section{Hemolysis ratio $(\%)=\frac{A_{s}-A_{N C}}{A_{P C}-A_{N C}} \times 100$}

Where, $A_{S}, A_{N C}$ and $A_{P C}$ are the absorbance values at $540 \mathrm{~nm}$ for supernatant in sample solution, negative control and positive control, respectively.

\section{Results And Discussion \\ 3.1. Reaction mechanism}

In the first step, $\mathrm{CMC}$ is oxidized in presence of sodium periodate that leads to ring cleavage at site with vicinal diols as well as oxidation (Step-I, Scheme 1). It helps in the formation of dialdehyde derivative of CMC. In a similar way, dialdehyde formation of dextrin takes place (Step-II, Scheme 1). Gelatin is used as such without any modification as performed in case of $\mathrm{CMC}$ and dextrin. The backbones (dialdehyde $\mathrm{CMC}$ and dextrin), when dissolved in presence of borax form hydrogen bonds using the $\mathrm{O}-\mathrm{H}$ groups left on dialdehyde $\mathrm{CMC}$ and dextrin with $\mathrm{O}-\mathrm{H}$ groups of tetrahydroxyl borate (formed by the hydrolysis of borax) (Step-I-II, Scheme 1). In a similar way, $\mathrm{N}-\mathrm{H}$ bonds present in gelatin form physical bonds with $\mathrm{O}-\mathrm{H}$ groups of tetra hydroxyl borate (Step-III, Scheme 1). The significance of using borax in all the solutions is that it acts as binding agent for the three backbones when they are mixed together. In this way, the dialdehyde CMC and gelatin as well as dialdehyde dextrin and gelatin come closer as tetrahydroxyl borate acts as binding agent for them. The aldehyde groups present in dialdehyde backbones form weak imide bonds with the gelatin backbone as aldehyde groups and $-\mathrm{NH}_{2}$ groups come closer to form imide linkage on dehydration. Finally, glutaraldehyde is added as crosslinking agent to find these bound polymeric chains with each other as the aldehyde groups present on it forms imide bonds with remaining $-\mathrm{NH}_{2}$ groups on gelatin backbone. Finally a complex structure with crosslinks is formed in the final hydrogel network (Scheme 2). Hence, the major bonding used in the network includes H-bonding and dynamic imide linkages (Shen et al., 2016; Maia, Carvalho, Coelho, Simões \& Gil, 2016; Pettignano et al., 2017; Balakrishnan, Joshi \& Banerjee, 2013; OU \& BO, 2017).

\subsection{Optimized of gelling time}

As we know, gelling time is the time taken for sol to gel conversion after addition of glutaraldehyde. It is based on the degree of crosslinking. Stronger the bonding between the chains, lesser is the gelling time. Various optimization parameters considered were: weight ratio of oxidized backbones: gelatin, concentration of borax, glutaraldehyde and amount of water.

It was found that minimum gelling time of 1 min was obtained at:1:1:10 weight ratio for $\mathrm{CMC}_{\mathrm{ox}}$ : Dex Gel, $0.021 \mathrm{~mol} / \mathrm{L}$ borax concentration, $6 \mathrm{ml}$ solvent and $0.035 \mathrm{~mol} / \mathrm{L}$ glutaraldehyde concentration.

In case of optimization of weight ratios of backbones, the weight ratio of oxidized backbones was kept fixed and that of gelatin was varied. It was found that with the increase in gelatin by weight with respect 
to fixed weight ratio of oxidized backbones, at first step, gelling time decreased and for next steps, increasing weight ratio w.r.t gelatin kept on increasing gelling time. It was so as in case of 1:1:10; the optimum weight ratio with minimum gelling time of $1 \mathrm{~min}$, the aldehyde groups were proportionate to $\mathrm{NH}_{2}$ groups present on gelatin. That was why, at first as the imide bonds start forming, the gelling time gets decreased (Supplementary data, Fig. 1a). However, above the optimum value, the gelatin weight ratio gives above the required number of $-\mathrm{NH}_{2}$ groups which remain non-bonding and increasing gelatin amount only hinders the process of sol-gel conversion by increasing the viscosity of medium (Sarkar et al., 2015; Balakrishnan \& Jayakrishnan, 2005).

The optimum borax concentration was found to be $0.021 \mathrm{~mole} / \mathrm{L}$ with gelling time of $2 \mathrm{~min}$. Firstly, increasing its concentration, increased the gelling time and after reaching a minimum value, it further starts increasing. This is due to the fact that at first stage, due to very low concentration borax is unable to bind the chains together. At optimum value, the binding agent concentration is most suitable for effective bonding among the chains. Above this optimum value, it starts hindering the process of imide bond formation as an effect of higher degree of binding through $\mathrm{H}$-bonding which hinders the process of gel formation (Supplementary data, Fig. 1b). Borax also provides the basic environment for imide bond formation (Balakrishnan, Joshi \& Banerjee, 2013; Balakrishnan \& Jayakrishnan, 2005).

Increasing the solvent amount also affects the gelling time. It first gets decreased as viscosity of solution containing backbones decreases that effectively let chains bind and crosslink together. However, above a certain optimum value, the gelling time increases as amount of water increases. It is so as the chains are getting far away and are now unable to bind and crosslink properly. The water molecules also try to solvate the different hydrophilic groups which does not let the chains interact with each other (Supplementary data, Fig. 1c). The minimum gelling time of 8 min was obtained for $6 \mathrm{ml}$ water (Balakrishnan, Joshi \& Banerjee, 2013; Sarkar et al., 2014; Balakrishnan \& Jayakrishnan, 2005).

Increasing glutaraldehyde concentration is also one of the most important factors affecting gelling time. At first, gelling time increases with increase in concentration of glutaraldehyde, as the amount is too small and non-homogenously distributed that is insufficient for crosslinking to occur. It then starts decreasing with increase in glutaraldehyde concentration, as glutaraldehyde starts forming imide bonds with the gelatin chains. It reaches a minimum at $0.035 \mathrm{~mole} / \mathrm{L}$ concentration with gelling time of $1 \mathrm{~min}$ (Supplementary data, Fig. 1d). Again, the gelling time started increasing with increase in glutaraldehyde concentration, as excessive concentration of it started interfering with the gelling process as the solution becomes viscous (Sarkar et al., 2014). The entire data has been given in Supplementary data, Table 1.

\subsection{Characterization techniques}

\subsubsection{SEM-EDS-EDS mapping}

SEM image of CMC, dextrin, their oxidized versions, gelatin and hydrogel were taken at same magnification. It was found that $\mathrm{CMC}$ has a fiber like structure. However, its dialdehyde derivative is no more a fiber, but it has a non-homogenous plain morphology with holes at some points (Fig. 1a, d). 
Dextrin however forms spherical particles in the same magnification range and its dialdehyde derivative is plain surface with cracks at some points (Fig. 1g, j). Gelatin has a clouded surface all over with holes and cracks somewhere (Fig. $1 \mathrm{~m}$ ). The hydrogel synthesized from the oxidized backbones and gelatin is porous surface with clearly visible pores of small as well as big size (Fig. 1p) (Priya et al., 2020)

EDS data was also covered in order to compare the elemental composition of various materials used as well as hydrogel. EDS data of CMC shows the presence of carbon (21.79 weight\% and 55.84 atomic\%), oxygen (18.34 weight\% and 35.29 atomic\%) and sodium ( 6.63 weight\% and 8.87 atomic\%) as it is polysaccharide unit with certain carboxylate groups bound to sodium ions. Dialdehyde CMC contains the same constituents with different weight\% and atomic\% values: carbon (14.82 weight\% and 52.42 atomic\%), oxygen (15.20 weight\% and 40.37 atomic\%) and sodium (3.90 weight\% and 7.21 atomic\%) (Fig. 1b, e). Dextrin is also a polysaccharide form having carbon and oxygen as basic compositional elements: carbon (61.09 weight\% and 67.65 atomic\%) and oxygen (38.91 weight\% and 32.35 atomic\%). Its oxidized form has the same elements with different weight and atomic percentage values such as carbon (52.47 weight\% and 59.52 atomic\%) and oxygen (47.53 weight\% and 40.48 atomic\%) (Fig. $1 \mathrm{~h}, \mathrm{k}$ ). Gelatin consisting of polypeptide based amide linkages and hence carbon ( 50.63 weight\% and 56.03 atomic\%), oxygen (24.37 weight\% and 20.24 atomic\%) and nitrogen (25.01 weight\% and 23.73 atomic\%) are the basic elements (Fig. 1n). The final hydrogel consist of imide linkages and borax based $\mathrm{H}$-bonding, hence the basic elements are carbon (51.49 weight\% and 53.14 atomic\%), oxygen (13.73 weight\% and 10.64 atomic\%), nitrogen (13.94 weight\% and 12.34 atomic\%) and boron (20.84 weight\% and 23.89 atomic\%) (Priya et al., 2020; Sharma et al., 2020) (Fig. 1q).

EDS mapping shows the similar type of elemental composition for each sample as the EDS data depicts. The elements have been colored (carbon as red, oxygen as green, sodium as yellow and boron as blue) and their distribution in the SEM images have been shown (Priya et al., 2020) (Fig. 1c, f, i, I, o, r).

\subsubsection{FT-IR}

FT-IR gives idea about the various functionalities present on the samples and clearly helps in distinguishing various samples. The characteristic IR stretching peaks found in case of $\mathrm{CMC}$ include a broad $\mathrm{O}-\mathrm{H}$ stretching at $3230 \mathrm{~cm}^{-1}, \mathrm{C}-\mathrm{H}$ stretching at $2891 \mathrm{~cm}^{-1}, \mathrm{C}=\mathrm{O}$ stretching at $1588 \mathrm{~cm}^{-1}$ and $\mathrm{C}-\mathrm{O}$ stretching at $1016 \mathrm{~cm}^{-1}$. This gives the evidence of polysaccharide moiety present there along with $\mathrm{O}-\mathrm{H}$ groups and carboxylate groups at side chains (Fig. 2a). It's dialdehyde version shows the same peaks at $3309 \mathrm{~cm}^{-1}$ (O-H stretch), $2947 \mathrm{~cm}^{-1}$ (C-H stretch), $1629 \mathrm{~cm}^{-1}$ (C = O) and $1038 \mathrm{~cm}^{-1}$ (C-O stretch). In addition to these peaks, a peak at $1728 \mathrm{~cm}^{-1}$ was found corresponding to aldehyde $\mathrm{C}=0$ stretching formed on ring cleavage which is a clear evidence of oxidation of the CMC sample (Balakrishnan, Joshi, Jayakrishnan \& Banerjee, 2014) (Fig. 2b). Similarly, dextrin show IR stretching frequencies at $3270 \mathrm{~cm}^{-}$ ${ }^{1}$ (O-H stretch), $2932 \mathrm{~cm}^{-1}$ (C-H stretch), $1645 \mathrm{~cm}^{-1}$ (C = O) and $995 \mathrm{~cm}^{-1}$ (C-O stretch). It shows that it forms a polysaccharide chain with O-H groups (Fig. 2C). The dialdehyde dextrin shows a peak at 1733 $\mathrm{cm}^{-1}$ in addition to peaks at $3313 \mathrm{~cm}^{-1}$ (O-H stretch), $2934 \mathrm{~cm}^{-1}$ (C-H stretch), $1619 \mathrm{~cm}^{-1}$ (C= O) and $1024 \mathrm{~cm}^{-1}$ (C-0 stretch) (Balakrishnan, Joshi, Jayakrishnan \& Banerjee, 2014). It forms the basis of 
evidence that oxidation of sample has been done to dialdehyde form on ring distortion (Fig. 2d). Gelatin consisting of amide bonds shows peaks at $3273 \mathrm{~cm}^{-1}$ (N-H stretch), $2937 \mathrm{~cm}^{-1}$ (C-H stretch), $1629 \mathrm{~cm}^{-1}$ $(\mathrm{C}=0), 1239 \mathrm{~cm}^{-1}$ (C-N stretch), $1529 \mathrm{~cm}^{-1}$ (N-H bending) and $1082 \mathrm{~cm}^{-1}$ (C-O stretch) which is a clear picture of polypeptide linkages (Fig. 2e). Hydrogel shows characteristic peaks at $3271 \mathrm{~cm}^{-1}(\mathrm{~N}-\mathrm{H}$ stretch), $2915 \mathrm{~cm}^{-1}$ (C-H stretch), $1638 \mathrm{~cm}^{-1}$ ( $\mathrm{C}=\mathrm{N}$ ), $1458 \mathrm{~cm}^{-1}$ (C-H bending), $1331 \mathrm{~cm}^{-1}, 1258 \mathrm{~cm}^{-1}$ (C-N stretch) and $1034 \mathrm{~cm}^{-1}$ (C-O stretch). Hence, the role of borax as well as glutaraldehyde is confirmed through the data. It is concluded that hydrogel was formed through $\mathrm{H}$-bonding with tetrahydroxyl borate units and imide bond formation among various carbonyl units and amine groups (Priya et al., 2020; Sharma et al., 2020; Sharma et al., 2019a; Priya et al., 2019; Sharma et al., 2019b; Sharma et al., 2019c; Talebian et al., 2019) (Fig. 2f).

\subsubsection{XPS survey}

XPS analysis is performed in order to determine the various types of elements and their type of bonding in the synthesized hydrogel. In a survey of intensity verses binding energy $(\mathrm{eV}) \mathrm{plot}$, it was clear that carbon $\left(\mathrm{C}_{1 \mathrm{~S}}\right)$, oxygen $\left(\mathrm{O}_{1 \mathrm{~S}}\right)$, nitrogen $\left(\mathrm{N}_{1 \mathrm{~S}}\right)$ and boron $\left(\mathrm{B}_{1 \mathrm{~S}}\right)$ were the main elements present in the hydrogel sample (Fig. 3a). Separate analysis of each element was also carried out. The XPS spectrum of carbon was plotted between intensity and binding energy $(\mathrm{eV})$ and peak fitting was also carried out. It was found to show peaks at $287.64 \mathrm{eV}, 285.80 \mathrm{eV}, 285.14 \mathrm{eV}, 283.80 \mathrm{eV}$ and $284.17 \mathrm{eV}$ corresponding to bonds $\mathrm{C}=0$ or $\mathrm{C}=\mathrm{N}, \mathrm{C}-\mathrm{N}, \mathrm{C}-\mathrm{O}, \mathrm{C}-\mathrm{C}$ and $\mathrm{C}=\mathrm{C}$, respectively. Similarly, XPS spectrum of nitrogen was analyzed after peak fitting and peaks were obtained at $400.00 \mathrm{eV}(\mathrm{N}-\mathrm{H}), 399.28 \mathrm{eV}(\mathrm{N}=\mathrm{C})$ and $398.90 \mathrm{eV}(\mathrm{N}-\mathrm{C})$. XPS spectrum of oxygen gives peaks at $532.94 \mathrm{eV}, 532.14 \mathrm{eV}$ and $531.41 \mathrm{eV}$, respectively for $\mathrm{C}=\mathrm{O}, \mathrm{O}-\mathrm{B}$ and $\mathrm{C}-\mathrm{O}$ bonds. However, in case of boron, only one peak was observed at $191.5 \mathrm{eV}$ for B-O bond (Sharma et al., 2020b; Sharma et al., 2019d) (Fig. 3b-e).

\subsection{XRD analysis}

The X-ray powder diffraction patterns (XRD) of all backbone and $\mathrm{CMC}_{\text {ox }}-\mathrm{Dex}_{\mathrm{ox}}$-Gel hydrogel are shown in Fig. 4a-e. From the data given in Supplementary data, Table 2, on oxidation of CMC, \% crystallinity was increased from $12.42 \%$ at $18.32^{\circ}$ to $29.20 \%$ at $22.50^{\circ}$ on $2 \theta$ scale. The intensity of the peaks were 1875.07 a.u. (d-spacing $=4.84 \mathrm{~A}^{\circ}, F W H M=0.36^{\circ}$, Crystalline size $\left.=0.29 \mathrm{~A}^{\circ}\right)$ and 172.38 a.u. $(\mathrm{d}$-spacing $=$ $3.95 A^{\circ}, F W H M=16.50^{\circ}$, Crystalline size $=0.36 A^{\circ}$ ), respectively. The \% crystallinity of dextrin was $70.29 \%$ at $18.92^{\circ}$ on $2 \theta$ scale with intensity 378.11 a.u. (d-spacing $=4.68 \mathrm{~A}^{\circ}$, FWHM $=22.03^{\circ}$, Crystalline size $=$ $0.30 \mathrm{~A}^{\circ}$ ). In oxidized dextrin, \% crystallinity was increased to $80.10 \%$ at $18.55^{\circ}$ on $2 \theta$ scale with intensity 296.14 (d-spacing $=4.78 \mathrm{~A}^{\circ}, F W H M=22.79^{\circ}$, Crystalline size $=0.29 \mathrm{~A}^{\circ}$ ). The $\%$ crystallinity of gelatin is $80.65 \%$ at $19.93^{\circ}$ on $2 \theta$ scale with intensity 435.96 a.u. (d-spacing $=4.45 A^{\circ}, F W H M=27.20^{\circ}$, Crystalline size $=0.32 \mathrm{~A}^{\circ}$ ). However, $\mathrm{CMC}_{\text {ox }}-\mathrm{Dex}_{\mathrm{ox}}$-Gel hydrogel is more crystalline in nature than the respective backbones as \% crystallinity was increased to $82.08 \%$ at $18.53^{\circ}$ on $2 \theta$ scale with intensity 318.16 a.u. (dspacing $=4.78 \mathrm{~A}^{\circ}, \mathrm{FWHM}=27.62^{\circ}$, Crystalline size $=0.29 \mathrm{~A}^{\circ}$ ). This is because of regular network of physical cross-linking ( $\mathrm{H}$-bonding and imide linkages) among the backbones, borax and glutaraldehyde throughout the polymeric structure of hydrogel (Sharma et al., 2020b; Sharma et al., 2019d) (Fig. 4). 


\subsection{Self-healing behavior}

It was also found out whether the hydrogel has the self-healing ability or not. Cylindrical as well as cubic samples were cut with knife from the middle. One of the cylindrical piece was kept in crystal violet dye for some time to give it a different coloration. Now, the knife cut samples were placed together for $4 \mathrm{~h}$ in contact with each other under pressure. After $4 \mathrm{~h}$, the samples were clearly visible to show self-healing behavior as the surfaces came in contact started forming broken dynamic imide bonds and $\mathrm{H}$-bonding interactions (Fig. 5), (Scheme 3) (Supplementary data). That results in the revival of the same hydrogel piece before cutting (Ginting, Pasaribu, Masmur, Kaban \& Hestina, 2020; Yang, Lu, Zhang \& Zhou, 2015; Chen et al., 2017).

\subsection{Rheological studies}

The hydrogel sample was placed between the plates and at increasing shear rate, it was allowed to be compressed. Clearly, the viscosity of the medium decreased with increase in shear rate. After certain value, it becomes consistent. Since, a non-linear curve has been obtained hence, it is the case of nonNewtonian gels. Viscosity decreases as a result of shear thinning. However, attaining a consistent behavior at the end is as a consequence of viscoelastic behavior of hydrogel. That means it can retain its shape after similar trend of decreasing the shear rate as it was increased. Since, the viscosity range for the sample is low enough (Fig. 6). Hence, it can be used in injectable hydrogels and for bio-printing (Chen et al., 2017; Zheng et al., 2018; Talebian et al., 2019). Supplementary data, Table 3 gives the comparative data of rheological studies with respect to other self-healing hydrogels in the market.

\subsection{In vitro degradation study}

The response of hydrogel immersed in various $\mathrm{pH}$ solutions, $0.1 \mathrm{M}$ phosphate buffer and $5 \mathrm{M}$ urea solutions was studied and it was found to show swelling as well as degradation initially as per \%weight increase along with yellow coloration of solution and finally the \% weight change also decreased till complete degradation within 6-7 days. It was observed in all the solutions. That means the hydrogel synthesized has the tendency to swell first and it gets degraded within 7 days. This is due to acidic as well as basic imine hydrolysis at different $\mathrm{pH}$ values. The PBS and urea solutions are taken up by the hydrogel and it gets swelled upto a certain limit and then starts getting degraded (Supplementary data, Table 4), (Supplementary data, Figs. 2-3). This is the clear evidence that physical interactions like Hbonding also exist in the hydrogel (Zhao et al., 2019).

\subsection{Blood compatibility test}

It was evaluated through hemolysis analysis whether the prepared hydrogel is blood compatible or not. The negative control (saline solution) possessing $0 \%$ hemolysis, positive control (distilled water)

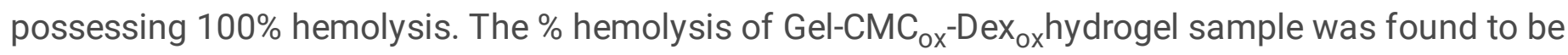
$3.59 \%$ which is less than permissible level of international standard $(<5 \%)$. This suggested the Gel$\mathrm{CMC}_{\text {ox }}$-Dex ox hydrogel is non-hemolytic in nature and it can be used for biomedical applications (Fig. 7) (Lei et al., 2019). 


\section{Conclusion}

Gelatin, dialdehyde dextrin, dialdehyde CMC were used to synthesize the self-healing hydrogels and borax and glutaraldehyde are used as binding agent and cross-linker, respectively at the room temperature. Gelatin with dialdehyde CMC, dialdehyde dextrin, and glutaraldehyde formed the imide bond and borax forms hydrogen bond with backbones, which gives the self-healing ability to the hydrogel. The optimized parameters for the preparation of $\mathrm{Gel}^{-} \mathrm{CMC}_{\mathrm{ox}}-\mathrm{Dex}_{\mathrm{ox}}$ hydrogel were dialdehyde $\mathrm{CMC}$, dialdehyde Dextrin, and Gelatin backbone in 1:1:10 ratio respectively, the cross-linkers borax $(0.021 \mathrm{~mol} / \mathrm{L})$, and glutaraldehyde $(0.035 \mathrm{~mol} / \mathrm{L})$, and $6 \mathrm{ml}$ solvent. The gel can degrade in $5 \mathrm{M}$ urea, Phosphate buffer, $\mathrm{pH}-$ $2.93,4.98,7.79,9.19$, and11.03 after 7th, 6th, 6th, 6th, 6th, 6th, and 6th days respectively. Hence, the hydrogel possesses biodegradable property. The blood compatibility test shows that the hemolysis ratio is $3.59 \%$. Hence, it has good blood compatibility because hemolysis ratio is less than the permissible international limits (5\%). Thus, it can be used in drug delivery and tissues engineering applications. The rheological studies give the average value of viscosity $(24,100 \mathrm{mPa}$. s), shear rate $(25.51 / \mathrm{s})$, and shear stress $(615 \mathrm{~Pa})$ after 63 second at the $25.1^{\circ} \mathrm{C}$ temperature. The Non-Newtonian properties are shown by the non-liner curve in the rheological graph. It shows that the hydrogel exhibits the shear-thinning properties. Hence, the apparent viscosity decreases with increase in the shear rate. This gives it injectable properties and worth being used in 3D printing.

\section{Declarations}

The authors declare no conflicts of interest. Studies involving human participants were in accordance with ethical standards.

\section{References}

Alvani, K., Qi, X., Tester, R. F. (2011). Use of carbohydrates, including dextrins, for oral delivery. Starch Stärke, 63 (7), 424-431.

Amaral, A. J. R., Emamzadeh, M., Pasparakis, G. (2018). Transiently malleable multi-healable hydrogel nanocomposites based on responsive boronic acid copolymers. Polymer Chemistry, 9 (4), 525-537.

Balakrishnan, B., Joshi, N., Banerjee, R. (2013). Borate aided Schiff's base formation yields in situ gelling hydrogels for cartilage regeneration. Journal of Materials Chemistry B, 1, 5564-5577.

Balakrishnan, B., Joshi, N., Jayakrishnan, A., Banerjee, R. (2014). Self-cross-linked oxidized alginate/gelatin hydrogel as injectable, adhesive biomimetic scaffolds for cartilage regeneration. Acta Biomaterialia, 10 (8), 3650-3663.

Balakrishnan, B., Joshi N., Thorat, K., Kaur, S., Chandan, R., Banerjee, R. (2019). A tumor responsive selfhealing prodrug hydrogel enables synergistic action of doxorubicin and miltefosine for focal combination chemotherapy. . Journal of Materials Chemistry B, 7, 2920-2925. 
Bergamasco, H., Marschel, C., Chadwick, B., Grady, F., Ma, M., Appel, E. A., Woo, Y. J. (2019). Use of a supramolecular polymeric hydrogel as an effective post-operative pericardial adhesion barrier. Nature Biomedical Engineering, 3 (8), 611-620.

Bhattacharyya, T., Chaudhuri, R., Das, K. S., Mondal, R., Mandal, S., Dash, J. (2019). Cytidine-Derived Hydrogels with Tunable Antibacterial Activities. ACS Appl. Bio Mater. 2019, 2, 3171-3177.

Boere, K. W. M., Blokzijl, M. M., Visser, J., Linssen, J. E. A., Malda, J., Hennink, W. E., Vermonden, T. (2015). Biofabrication of reinforced 3D-scaffolds using two-component hydrogels. Journal of Materials Chemistry B, 3 (46), 9067-9078.

Buhus, G., Popa, M., Desbrieres, J. (2009). Hydrogels Based on Carboxymethylcellulose and Gelatin for Inclusion and Release of Chloramphenicol. Journal of Bioactive and Compatible. Polymers, 24 (6), 525545 .

Cao, Y., Morrissey, T. G., Acome, E., Allec, S. I., Wong, B. M., Keplinger, C., Wang, C. A. (2017). Transparent, Self-Healing, Highly Stretchable Ionic Conductor. Advanced Materials, 29 (10), 1605099, 1-9.

Chen, J., Peng, Q., Thundat, T., Zeng, H. (2019). Stretchable, Injectable, and Self-Healing Conductive Hydrogel Enabled by Multiple Hydrogen Bonding toward Wearable Electronics. Chemistry of Materials, 31, 4553-4563.

Chen, M. H., Wang, L. L., Chung, J. J., Kim, Y.H., Atluri, P., Burdick, J. A. (2017). Methods To Assess ShearThinning Hydrogels for Application As Injectable Biomaterials. ACS Biomaterials Science \& Engineering, 3 (12), 3146-3160.

Dakin, H. D. (1920). Amino-acids of gelatin. The Journal of Biological Chemistry, 44, 499-529.

Ginting, M., Pasaribu, S.P., Masmur, I., Kaban, J., Hestina (2020). Self-healing composite hydrogel with antibacterial and reversible restorability conductive properties. RSC Advances, 10 (9), 5050-5057.

Grosskopf, A. K., Roth, G. A., Smith, A. A. A., Gale, E. C., Hernandez, H. L, Appel, E. A. (2019). Injectable supramolecular polymer-nanoparticle hydrogels enhance humanmesenchymal stem cell delivery. Bioengineering \& Translational Medicine, 5 (1), 10147, 1-11.

Hollabaugh, C. B., Burt, L. H., Walsh, A. P. (1945). Carboxymethyl cellulose. Uses and Applications. Industrial \& Engineering Chemistry, 37 (10), 943-947.

Holten-Andersen, N., Harrington, M. J., Birkedal, H., Lee, B. P., Messersmith, P. B., Lee, K. Y. C., Waite, J. H. (2011). pH-Induced Metal Ligand Cross-Links Inspired by Mussel Yield Self-Healing Polymer Networks with near-Covalent Elastic Moduli. Proceedings of the National Academy of Sciences of the United States of America, 108 (7), 2651-2655. 
Jalalvandi, E., Shavandi, A. (2018). Shear thinning/self-healing hydrogel based on natural polymers with secondary photocrosslinking for biomedical applications. Journal of the Mechanical Behavior of Biomedical Materials, 90, 191-201.

Khamrai, M., Banerjee, S. L., Paul, S., Samanta, S., Kundu, P. P. (2019). Curcumin entrapped gelatin/ionically modified bacterial cellulose based self-healable hydrogel film: An eco-friendly sustainable synthesis method of wound healing patch. International Journal of Biological Macromolecules, 122, 940-953.

Lai, J. C., Jia, X. Y., Wang, D. P., Deng, Y. B., Zheng, P., Li, C. H., Zuo, J. L., Bao, Z. (2019).

Thermodynamically stable whilst kinetically labile coordination bonds lead to strong and tough selfhealing polymers. Nature Communications, 10, 1164.

Lei, J., Li, X., Wang, S., Yuan, L., Ge, L., Li, D., Mu, C. (2019). Facile Fabrication of Biocompatible GelatinBased Self-Healing Hydrogels. ACS Applied Polymer Materials, 1 (6), 1350-1358.

Maeda, T., Otsuka, H., Takahara, A. (2009). Dynamic Covalent Polymers: Reorganizable Polymers with Dynamic Covalent Bonds. Progress in Polymer Science, 34 (7), 581-604.

Maia, J., Carvalho, R. A., Coelho, J. F. J., Simões, P. N., Gil, M. H. (2016). Insight on the periodate oxidation of dextran and its structural vicissitudes. Polymer, 52 (2), 258-265.

Mariod, A. A., Adam, H. F. (2013). Review: gelatin, source, extraction and industrial applications. Acta Scientiarum Polonorum, Technologia Alimentaria, 12 (2), 135-147.

Naghizadeh, Z., Karkhaneh, A., Khojasteh, A. (2018). Self-crosslinking effect of chitosan and gelatin on alginate based hydrogels: injectable in situ forming scaffolds. Materials Science \& Engineering C, 89, 256264.

Narkar, A. R., Lee, B. P. (2018). Incorporation of Anionic Monomer to Tune the Reversible CatecholBoronate Complex for pH-Responsive, Reversible Adhesion. Langmuir, 34 (32), 9410-9417.

OU, A.; BO, I. (2017). Chitosan Hydrogels and their Glutaraldehyde-Crosslinked Counterparts as Potential Drug Release and Tissue Engineering Systems - Synthesis, Characterization, Swelling Kinetics and Mechanism. Journal of Physical Chemistry \& Biophysics, 07 (03), 1000256-1-1000256-7.

Ouyang, L., Highley, C. B., Rodell, C. B., Sun, W., \& Burdick, J. A. (2016). 3D Printing of Shear-Thinning Hyaluronic Acid Hydrogels with Secondary Cross-Linking. ACS Biomaterials Science \& Engineering, 2 (10), $1743-1751$.

Pettignano, A., Häring, M., Bernardi, L., Tanchoux, N., Quignard, F., DíazDíaz, D. (2017). Self-healing alginate-gelatin biohydrogels based on dynamic covalent chemistry: elucidation of key parameters. Materials Chemistry Frontiers, 1(1), 73-79. 
Pourjavedi, A., Soleyman, R., Bardajee, G. R., Ghavami, S. (2009). Novel superabsorbent hydrogel based on natural hybrid backbone: Optimized synthesis and its swelling behavior. Bulletin of the Korean Chemical Society, 30, 2680-2686.

Priya, Kaith, B.S., Shanker, U., Gupta, B., Bhatia, J. K. (2018). RSM-CCD optimized In-air synthesis of photocatalyticnanocomposite: Application in removal-degradation of toxic brilliant blue. Reactive and Functional Polymers, 131, 107-122.

Priya, Sharma, A. K., Kaith, B. S., Vipula, Chandel, K., Singh, A., Isha (2020). Chemically modified chitosan-sodium alginate as chemo-sensor adsorbent for the detection of picric acid and removal of biebrich scarlet. International Journal of Biological Macromolecules, 147, 582-594.

Priya, Sharma, A. K., Kaith, B. S., Tanwar, V., Bhatia, J. K., Sharma, N., Bajaj, S., Panchal, S. (2019). RSM CCD Optimized Sodium Alginate/Gelatin Based ZnS -Nanocomposite Hydrogel for the Effective Removal of Biebrich Scarlet and Crystal Violet Dyes. International Journal of Biological Macromolecules, 129, 214 $-226$.

Sarker, B., Papageorgiou, D. G., Silva, R., Zehnder, T., Gul-E-Noor, F., Bertmer, M., Kaschta, J., Chrissafis, K., Detsch, R., Boccaccini, A. R. (2014). Fabrication of alginate-gelatin crosslinked hydrogel microcapsules and evaluation of the microstructure and physico-chemical properties. Journal of Materials Chemistry $\mathrm{B}$, 2 (11), 1470-1482.

Shalviri, A., Liu, Q., Abdekhodaie, M. J., Wu, X. Y. (2010). Novel modified starch-xanthan gum hydrogels for controlled drug delivery: Synthesis and characterization. Carbohydrate Polymers, 79, 898-907.

Sharma, A. K., Kaith, B.S., Gupta, B., Shanker, U., Lochab, S.P. (2018). A facile strategy to synthesize a novel and green nanocomposite based on gum Salaiguggal-Investigation of antimicrobial activity. Materials Chemistry and Physics, 219, 129-141.

Sharma, A. K., Priya, Kaith, B. S., Bajaj, S., Bhatia, J.K., Panchal, S., Sharma, N., Tanwar, V. (2019a). Efficient capture of eosin yellow and crystal violet with high performance xanthan -acacia hybrid super adsorbent optimized using response surface methodology. Colloids Surfaces B: Biointerfaces, 175, 314 323.

Sharma, A. K., Priya, Kaith, B. S., Isha., Singh, A., Chandel, K., Vipula (2020). Riboflavin Functionalized Dextrin -Sodium Alginate Based Fluorescent Sensor: Detoxification of $\mathrm{Cu} 2+$ and $\mathrm{Ni} 2+$ Ions. ACS Applied Polymer Materials, 1 (11), 3084 -3094.

Sharma, A. K., Priya, Kaith, B.S., Panchal, S., Bhatia, J. K., Bajaj, S., Tanwar, V., Sharma, N. (2019b). Response surface methodology directed synthesis of luminescent nanocomposite hydrogel for trapping anionic dyes. Journal of Environmental Management, 231, 380-390. 
Sharma, A. K., Priya, Kaith, B. S., Sharma, N., Bhatia, J.K., Tanwar, V., Panchal, S., Bajaj, S. (2019c). Selective removal of cationic dyes using response surface methodology optimized gum acacia -sodium alginate blended superadsorbent. International Journal of Biological Macromolecules, 124, 331 -345.

Sharma, A. K., Priya, Kaith, B. S., Singh, A., Isha, Vipula, Chandel, K. (2019d). Enzymatic construction of quinine derivative of dextrin/PVA based hybrid gel film for the simultaneous detection and removal of copper and lead ions in real water samples. Chemical Engineering Journal, 122891.

Shen, X., Yang, M., Cui, C., Cao, H. (2019). In situ immobilization of glucose oxidase and catalase in a hybrid interpenetrating polymer network by 3D bioprinting and its application. Colloids and Surfaces A: Physicochemical and Engineering Aspects, 568, 411-418.

Shen, Y., Li, X., Huang, Y., Chang, G., Cao, K., Yang, J., Ye, X. (2016). pH and redox dual stimuli-responsive injectable hydrogels based on carboxymethyl cellulose derivatives. Macromolecular Research, 24 (7), 602-608.

Sherrington, D. C., Taskinen, K. A. (2001). Self-Assembly in Synthetic Macromolecular Systems via Multiple Hydrogen Bonding Interactions. Chemical Society Reviews, 30 (2), 83-93.

Shi, Y., Wang, M., Ma, C., Wang, Y., Li, X., Yu, G. A. (2015). Conductive Self-Healing Hybrid Gel Enabled by Metal-Ligand Supramolecule and Nanostructured Conductive Polymer. Nano Letters, 15 (9), 6276-6281.

Stapleton, L. M., Steele, A. N., Wang, H., Lopez Hernandez, H., Yu, A. C., Paulsen, M. J., Smith, A. A. A., Roth, G. A., Thakore, A. D., Lucian, H. J., Totherow, K. P., Baker, S. W., Tada, Y., Farry, J. M., Eskandari, A., Hironaka, C. E., Jaatinen, K. J., Williams, K. M., Balakrishnan, B., Jayakrishnan, A. (2005). Self-crosslinking biopolymers as injectable in situ forming biodegradable scaffolds. Biomaterials, 26 (18), $3941-$ 3951.

Talebian, S., Mehrali, M., Taebnia, N., Pennisi, C., P., Kadumudi, F. B., Foroughi, J., Hasany, M., Nikkhah, M., Akbari, M., Orive, G., Dolatshahi-Pirouz, A. (2019). Self-Healing Hydrogels: The Next Paradigm Shift in Tissue Engineering. Advanced Science, 6 (16), 1801664, 1-47.

Tu,Y., Chen, N., Li, C., Liu, H., Zhu, R., Chen, S., Xiao, Q., Ramakrishna, S.; He, L. (2019). Advances in Injectable Self-healing Biomedical Hydrogels. Acta Biomaterialia, 90, 1-20.

Tuncaboylu, D. C., Sari, M., Oppermann, W., Okay, O. (2011). Tough and Self-Healing Hydrogels Formed Via Hydrophobic Interactions. Macromolecules, 44(12), 4997-5005.

Wang, H., Yang, Y., Nishiura, M., Higaki, Y., Takahara, A., Hou, Z. (2019). Synthesis of Self-Healing Polymers by Scandium-Catalyzed Copolymerization of Ethylene and Anisylpropylenes. Journal of the American Chemical Society, 141 (7), 3249-3257.

Yang, L., Lu Lu, Zhang, C., Zhou, C. (2015). Highly Stretchable and Self-healing Hydrogels Based on Poly(acrylic acid) and Functional POSS. Chinese Journal of Polymer Science, 34 (2), 185-194. 
Zhao, Q., Mu, S., Long, Y., Zhou, J., Chen, W., Astruc, D., Gaidau, C., Gu, H. (2019). Tannin-Tethered Gelatin Hydrogels with Considerable Self-Healing and Adhesive Performances. Macromolecular Materials and Engineering, 1800664, 1-8.

Zheng, Y., Liang, Y., Zhang, D., Sun, X., Liang, L., Li, J., Liu, Y.N. (2018). Gelatin-Based Hydrogels Blended with Gellan as an Injectable Wound Dressing. ACS Omega, 3, 4766-4775.

Zhivkov, A. M., Hristov, R. P. (2015). Adsorption of carboxymethyl cellulose on alumina particles. Journal of Colloid and Interface Science, 447, 159-166.

\section{Figures}



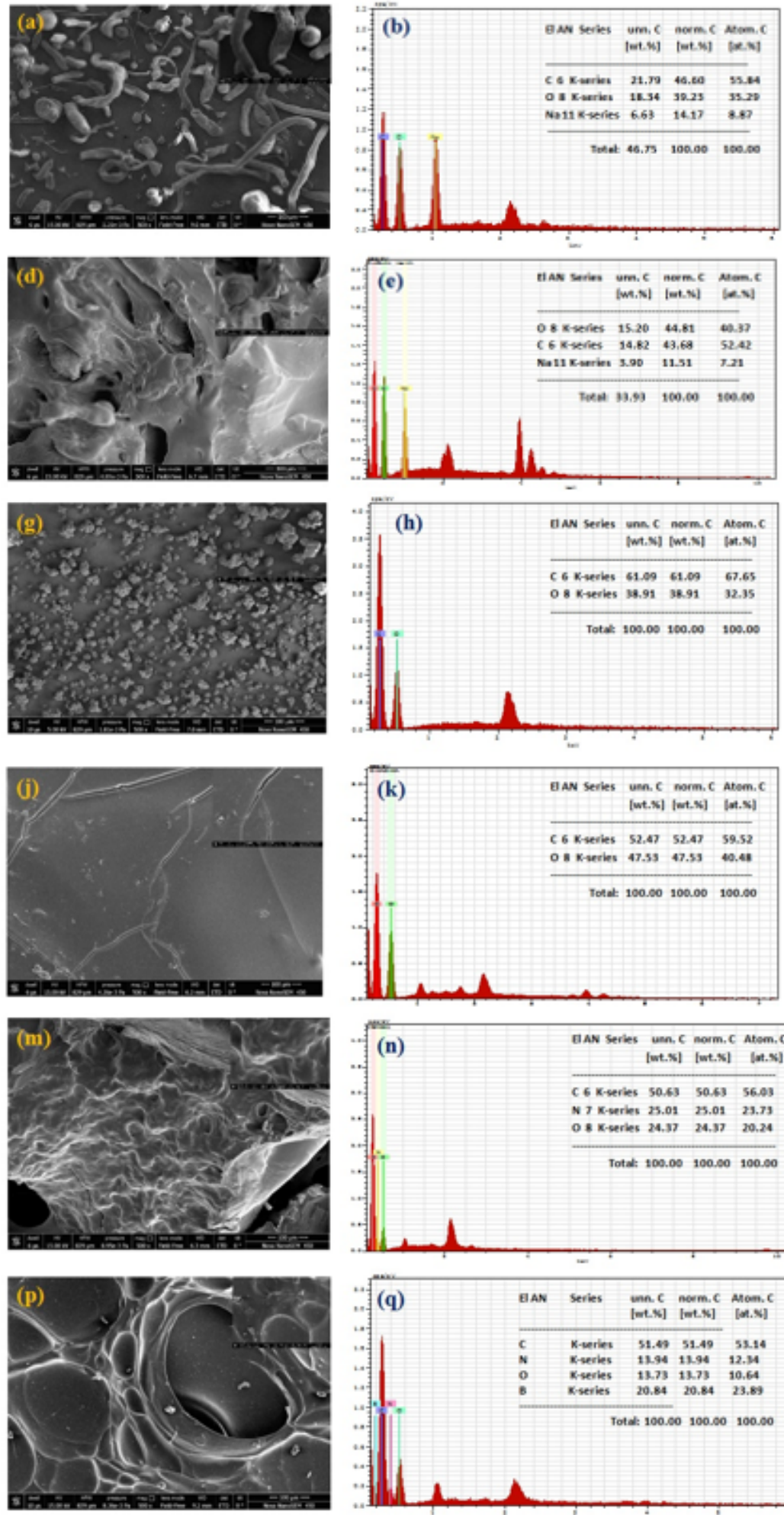
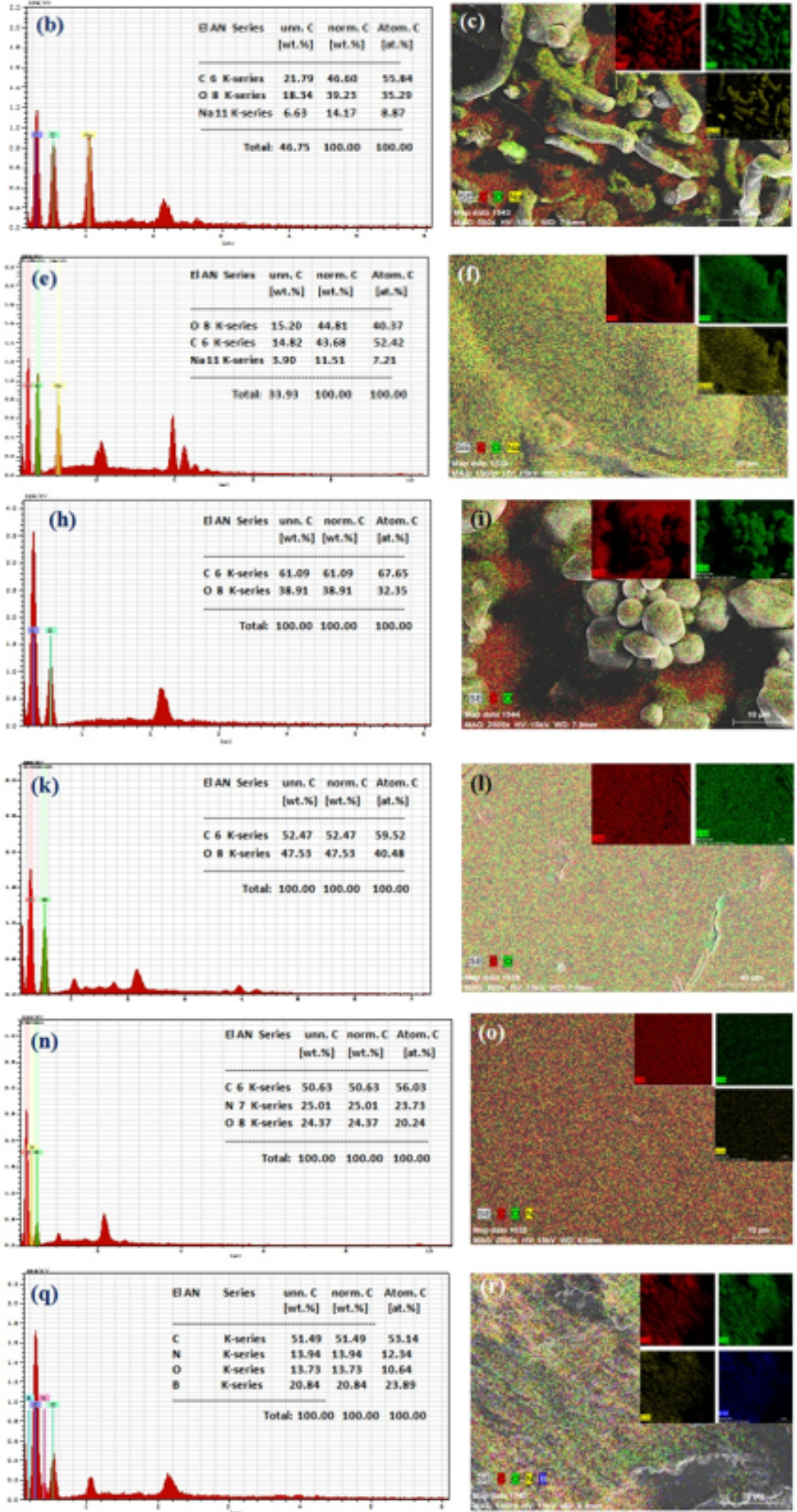

\section{Figure 1}

SEM-EDS-EDS mapping: Carboxymethyl cellulose (a-c), Oxidized Carboxymethyl cellulose (d-f), Dextrin (gi), Oxidized Dextrin (j-l), Gelatin (m-o); Gel-CMCox-Dexoxhydrogel ( $p-r)$ 


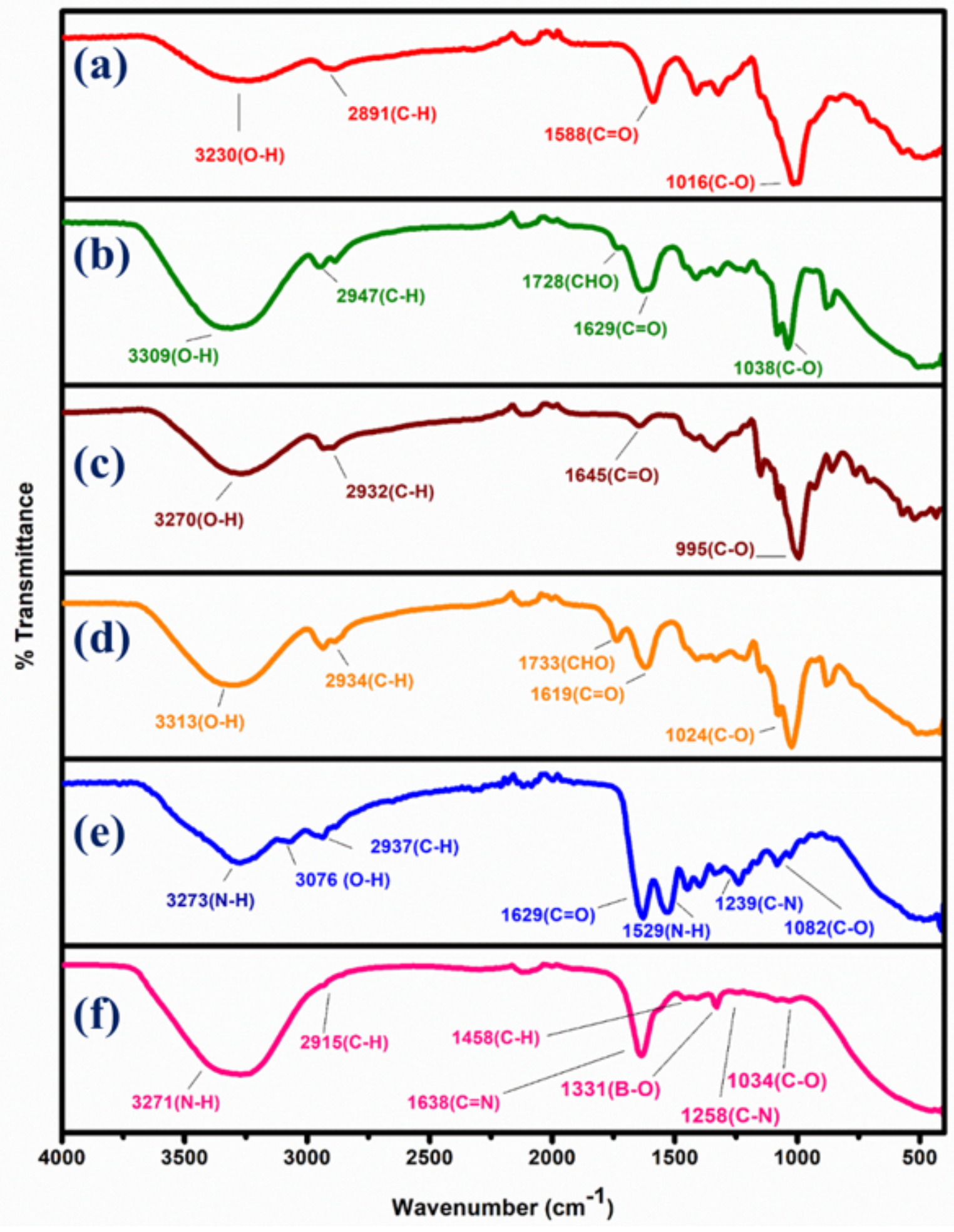

Figure 2

IR spectrum of (a) Carboxymethyl cellulose (b) Oxidized Carboxymethyl cellulose (c) Dextrin (d) Oxidized Dextrin (e) Gelatin (f) Gel-CMCox-Dexox hydrogel 

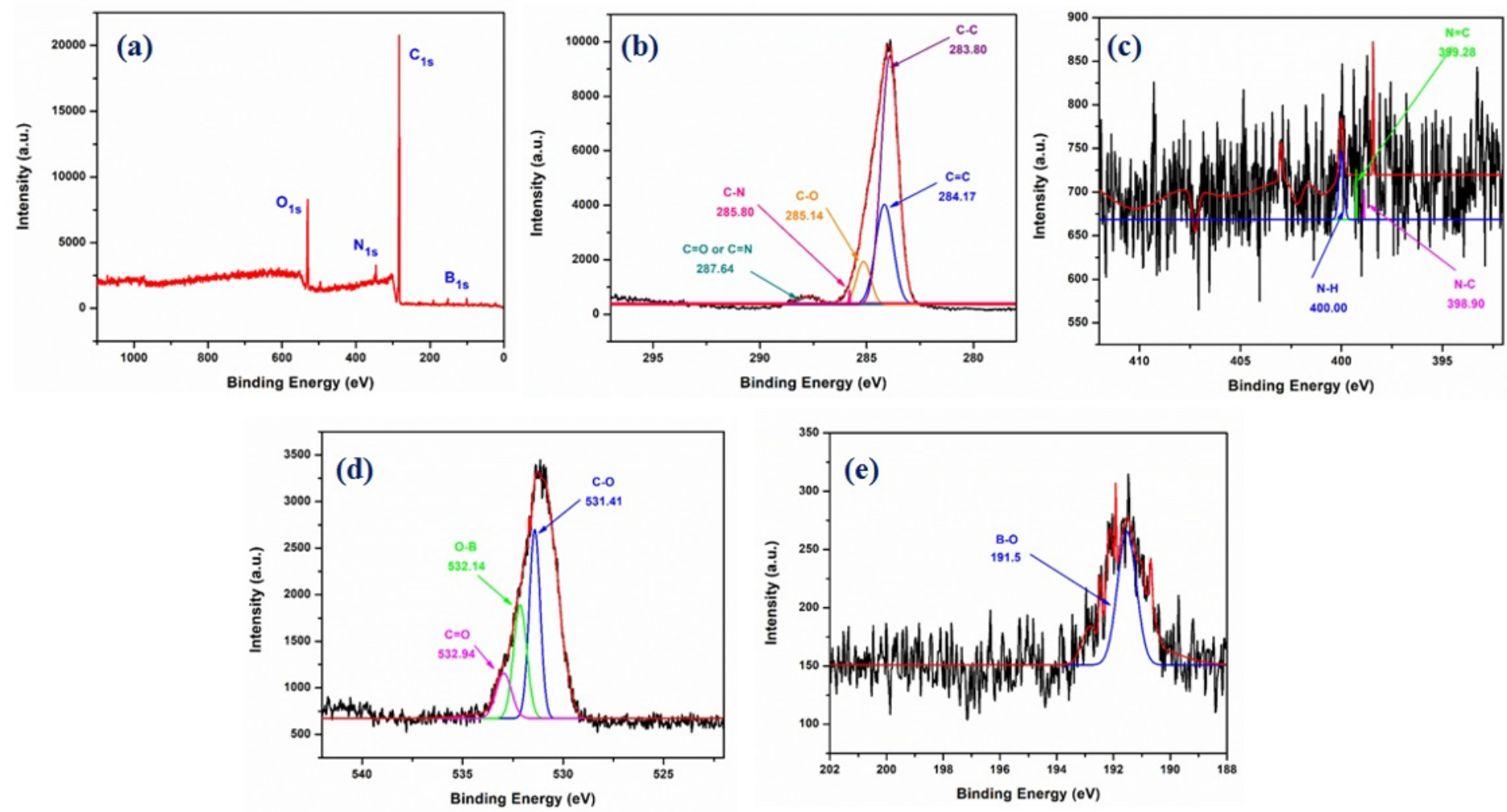

Figure 3

XPS analysis survey of (a) Gel-CMCox-Dexoxhydrogel; XPS spectrum of (b) carbon (c) nitrogen (d) oxygen (e) boron 


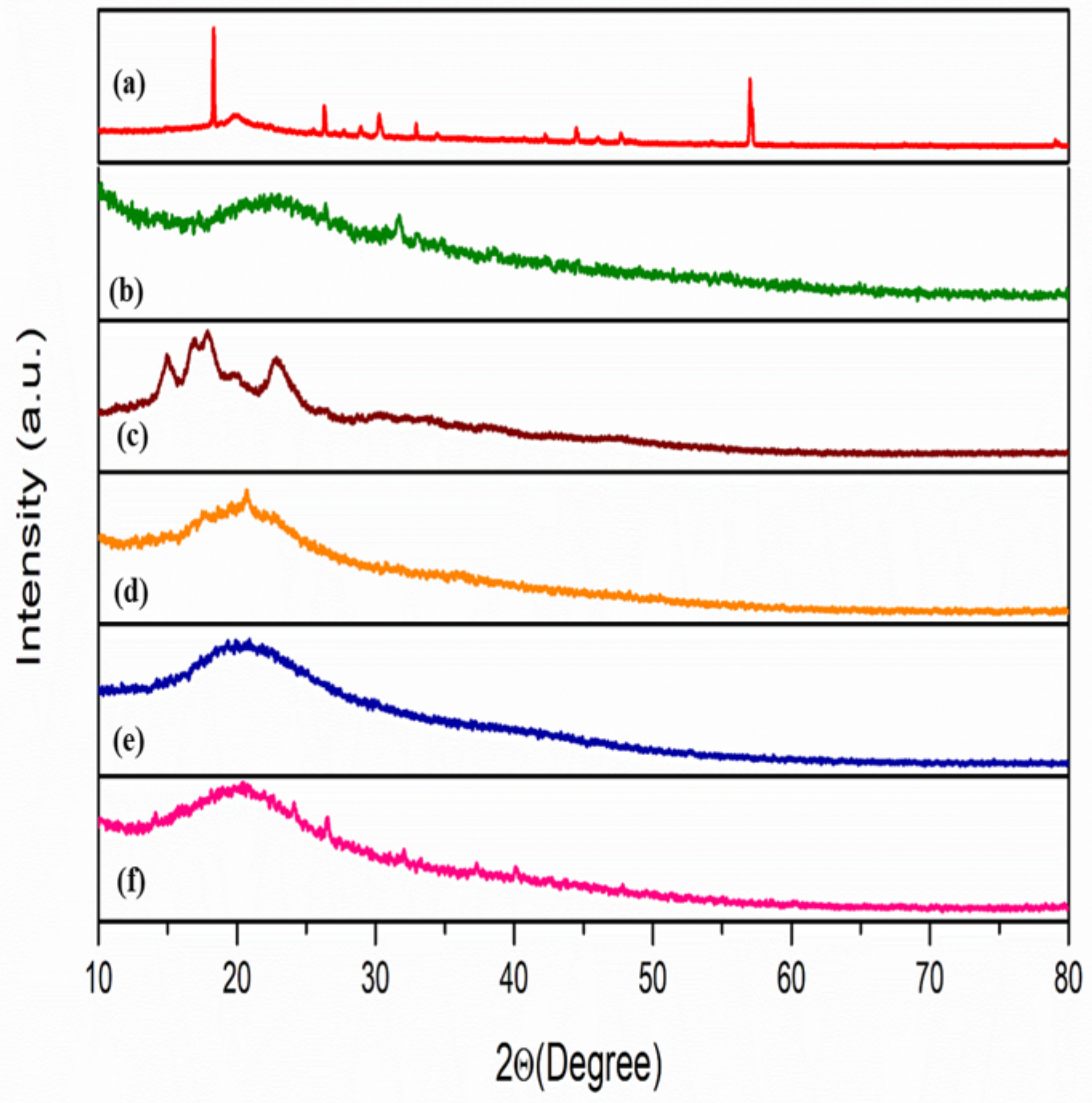

Figure 4

XRD spectra of (a) Carboxymethyl cellulose (b) Oxidized Carboxymethyl cellulose (c) Dextrin (d) Oxidized Dextrin (e) Gelatin (f) Gel-CMCox-Dexox hydrogel 

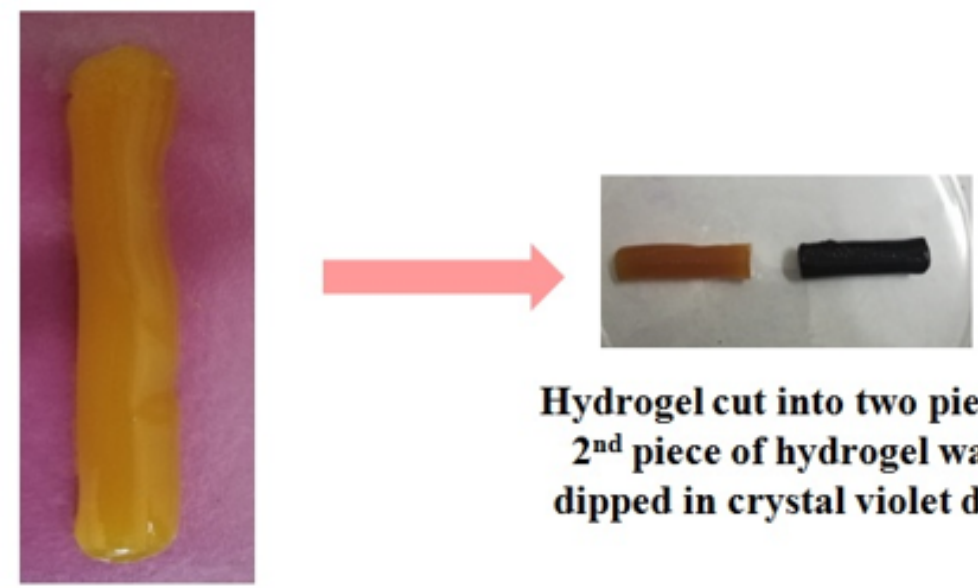

Self-Healing

After 4 h

\section{Hydrogel cut into two pieces} $2^{\text {nd }}$ piece of hydrogel was dipped in crystal violet dye

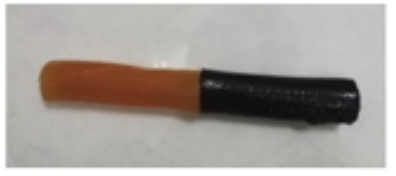

Self-healing of two pieces of Hydrogel

\section{Hydrogel in}

\section{Cylindrical form}

Figure 5

Self-healing behavior of Gel-CMCox-Dexox hydrogel

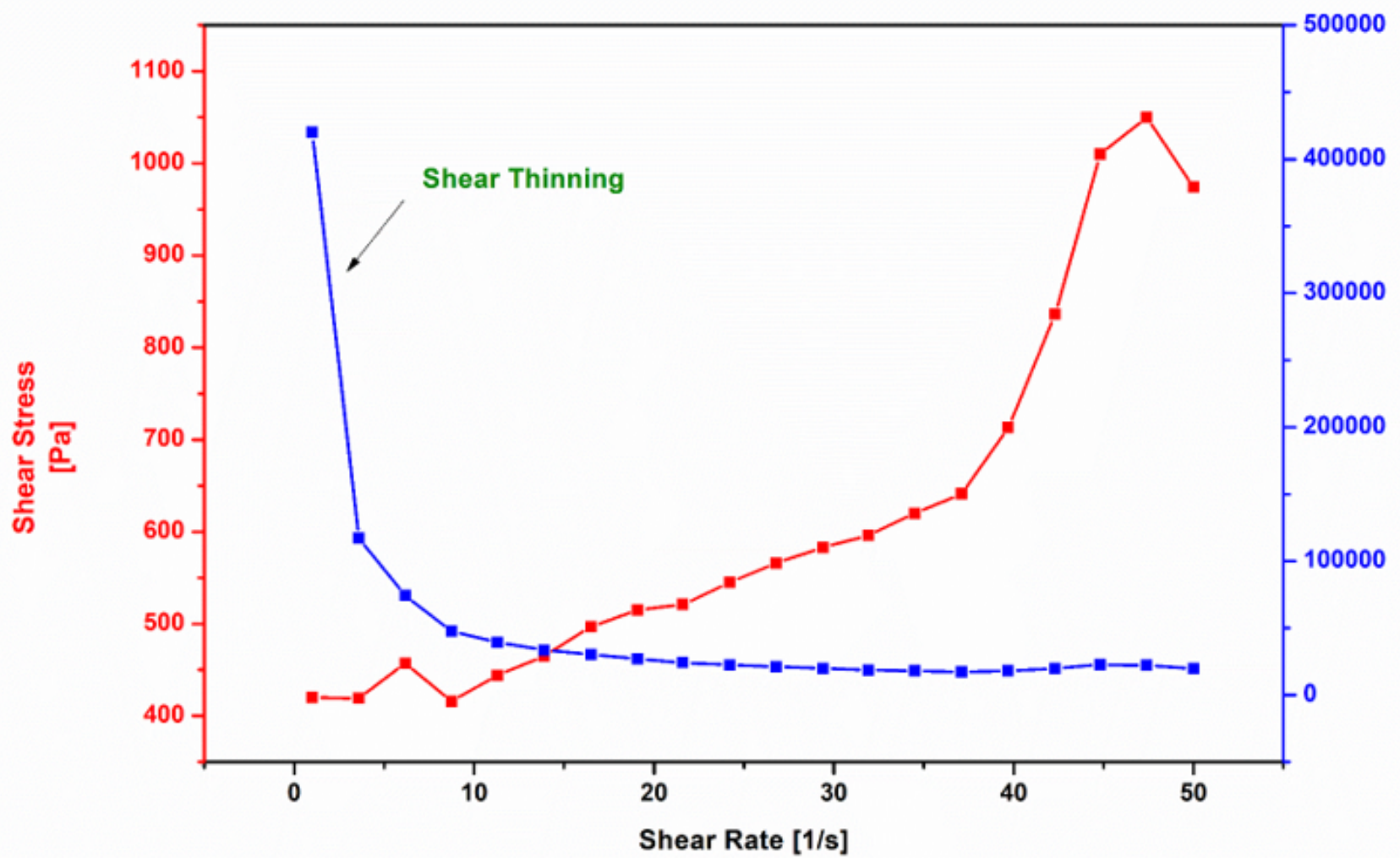

Figure 6

Viscosity v/s shear rate and shear stress v/s shear rate graph of Gel-CMCox-Dexox hydrogel 


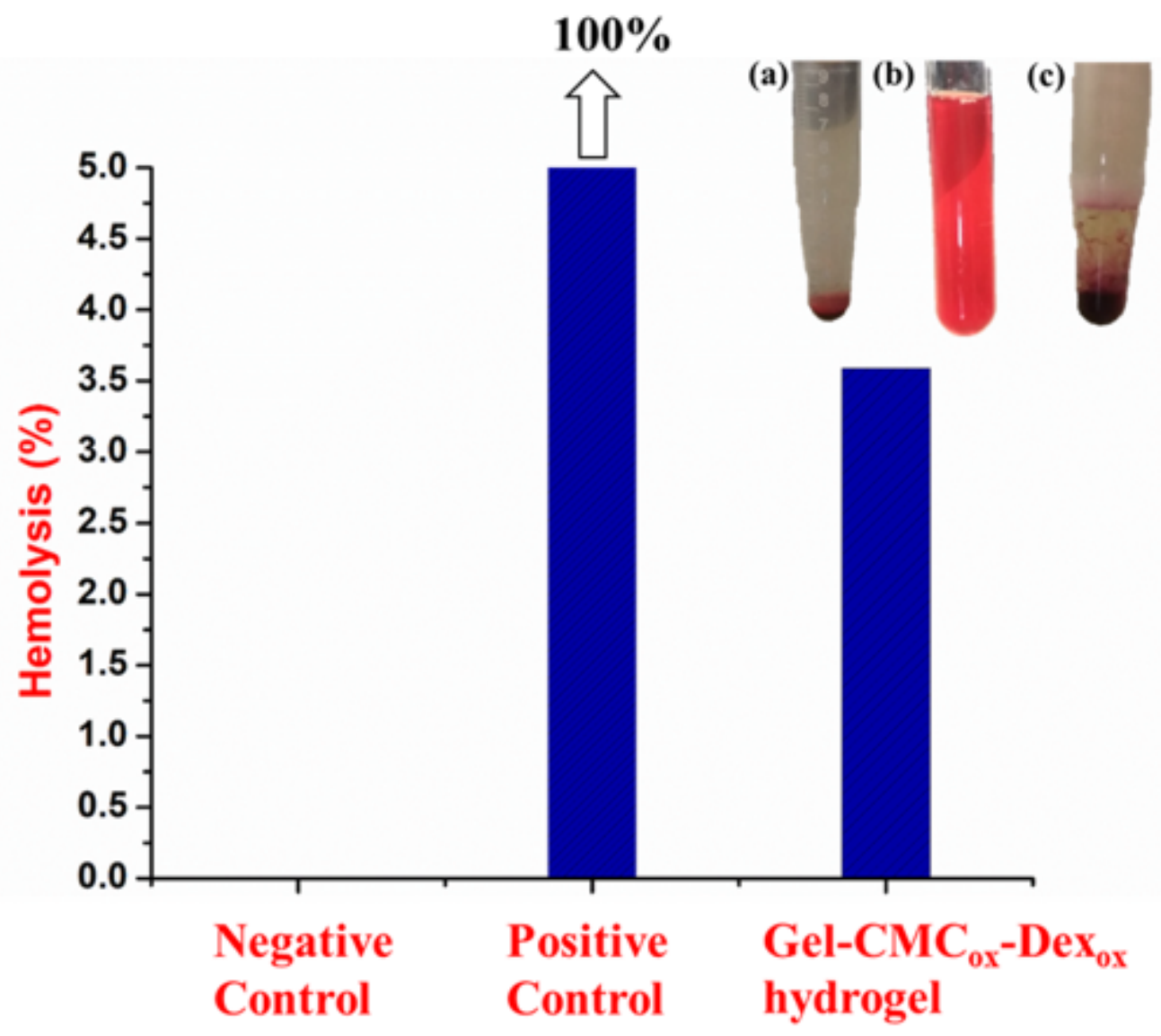

Figure 7

(a) Blood in saline solution as negative control (b) Blood in water as positive control (c) Blood in GelCMCox-Dexoxhydrogel 


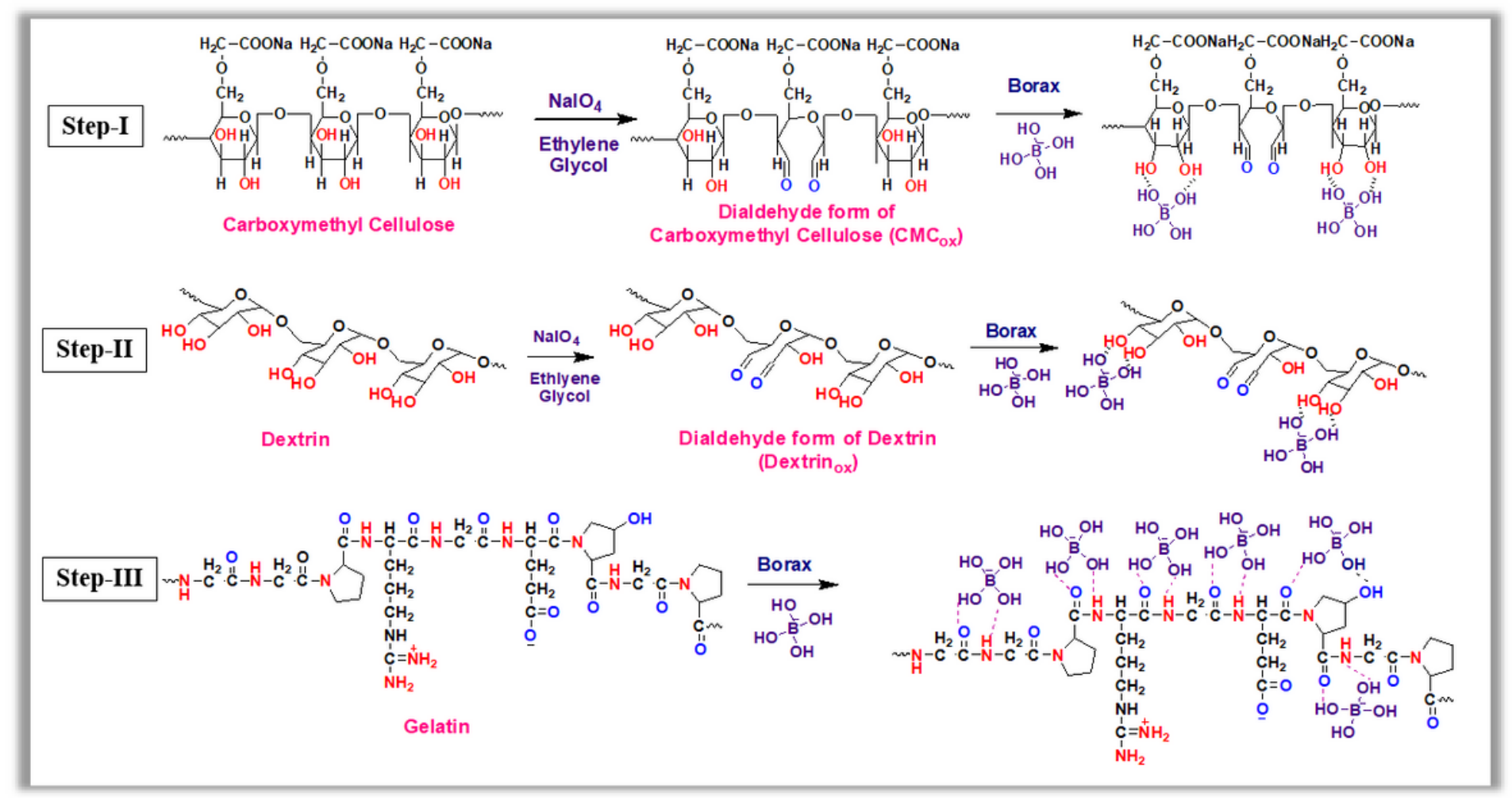

\section{Figure 8}

Scheme 1 Periodate oxidation of CMC and its bonding with borax (Step-I), Periodate oxidation of Dextrin and its bonding with borax (Step-II), Bonding of Gelatin with borax (Step-III) 


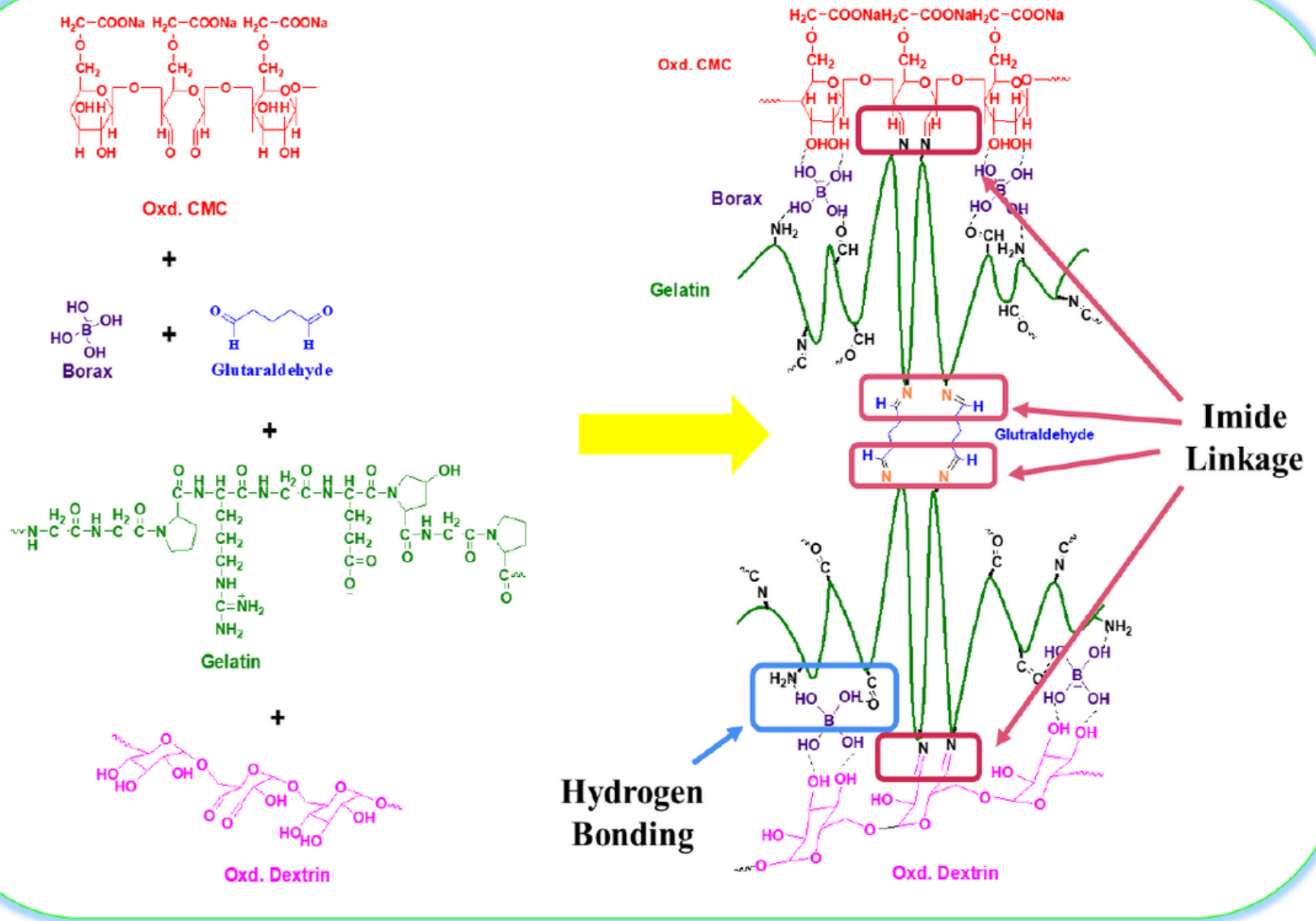

\section{Figure 9}

Scheme 2 Reaction mechanism for the synthesis of Gel-CMCox-Dexox hydrogel
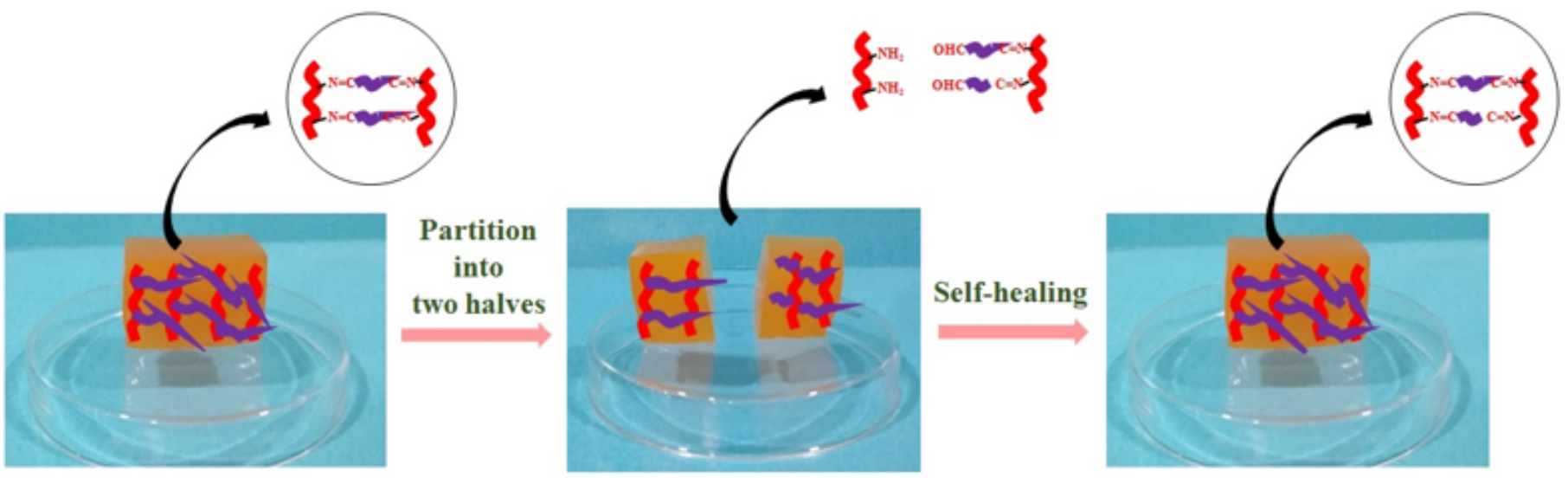

Figure 10

Scheme 3 Self-healing mechanism of Gel-CMCox-Dexoxhydrogel 


\section{Supplementary Files}

This is a list of supplementary files associated with this preprint. Click to download.

- SUPPLEMENTARYDATA.docx 